\title{
LA LÈPRE
}

médecine/sciences $1987 ; 3: 442-3$

\section{Philippe $\mathbf{H}$. Lagrange}

Professeur de microbiologie à l'université Pierreet-Marie-Curie Chef du laboratoire de microbiologie, Hôtel-Dieu

\section{ADRESSE}

$\mathrm{Ph}$. H. Lagrange : laboratoire de microbiologie, Hôtel-Dieu, 1, place du Parvis-Notre-Dame,

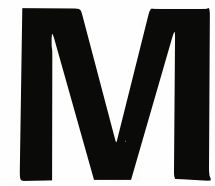

aladie ancestrale, la lèpre atteint encore, en 1987, environ 15 millions d'individus vivant surtout dans les pays en voie de développement (un lépreux sur trois vit en Inde).

Maladie énigmatique, elle interpelle encore de nos jours les bactériologistes, les immunologistes, les épidémiologistes et les cliniciens du fait d'un certain nombre de questions non encore résolues.

La première particularité de cette maladie infectieuse transmissible concerne l'agent pathogène ; si celui-ci (Mycobacterium leprae) a été parmi les premiers décrits comme agent responsable d'une maladie transmissible, par G.H. Armauer Hansen, à Bergen (Norvège), en 1873, tous les efforts réalisés depuis cette date pour le cultiver in vitro dans des milieux acellulaires ont échoué. Quelques essais ont néanmoins montré la possibilité de survie et de croissance limitée dans des cultures cellulaires humaines (macrophages, cellules de Schwann, fibroblastes), mais peu compatible avec des études biochimiques et moléculaires de quelque envergure. Par ailleurs, près de cent ans se sont écoulés entre la reconnaissance de $M$. leprae et l'établissement du premier modèle expérimental animal, permettant la multiplication du bacille de Hansen in vivo. En effet, c'est en 1960 que Shepard, le premier, a décrit les conditions expérimentales permettant la multiplication de $M$. leprae dans le coussinet plantaire de la souris [1]. C'est ainsi que l'on a pu démontrer le rôle joué par la réponse immunitaire thymodépendante [2] dans l'acquisition de la résistance infectieuse des souris et comparer les propriétés des différentes souches isolées dans le monde, sans montrer de réelle différence suivant leur origine géographique. De plus, ce modèle a permis de définir certaines caractéristiques biologiques de $M$. leprae : temps de doublement, temps de survie en fonction de la température, agents inactivants. Il est actuellement régulièrement utilisé pour évaluer l'efficacité in vivo de nouveaux antibiotiques contre le bacille de Hansen et pour tester la sensibilité des bactéries isolées des lésions vis-à-vis des antibiotiques utilisés. Cette dernière approche a révélé l'ampleur mondiale de la résistance de $M$. leprae visà-vis de la Dapsone. Enfin, cette méthode permet de juger de la valeur de certaines mesures prophylactiques vaccinales (BCG par exemple) [3]. Un autre modèle animal a été développé, en 1971, par Kircheimer et Storrs [4]. L'injection de $M$. leprae $\left(1 \times 10^{6}\right.$ à $1 \times 10^{7}$ /animal) chez certains tatous à neuf ban-

\section{RÉFÉRENCES}

1. Shepard CC. The experimental disease that follows the injection of human leprosy bacilli into foot-pads of mice. $J$ Exp Med 1960 ; 112 : 445-54.

2. Rees JWR. Enhanced susceptibility of thymectomised and irradiated mice to infection with Mycobacterium leprae. Nature 1966 ; 211: 657-8.

3. Rees RJW. The contribution of Charles C. Shepard to leprosy research : from the mouse footpad model to new DNA technology. Lepr Rev 1986 ; 57 (suppl. 2) : 1-14

4. Kirchheimer WF, Storrs EE. Attemps to establish the armadillo (Dasypus novimcinctus Linn) as a model for the study of leprosy : I Report of lepromatoïd leprosy in experimentally infected armadillo. Int $J$ Lepr 1971; 39 : 693-701.

5. Clark-Curtin JE, Jacobs WR, Docherty MA, Ritchie LR, Curtis R. Molecular analysis of DNA and construction of genomic libraries of Mycobacterium leprae. J Bacteriol 1985 ; 161 : 1093-102. 6. Ridley DS, Jopling WH. Classification of leprosy according to immunity: A five groups system. Int $J$ Lepr 1986; 34 : 255-73.

7. Brennan PJ, Barrow WW. Evidence for species specific lipid antigens in Mycobacterium leprae. Int J Lepr 1980 ; 48 : 382-7.

8. Mehra V, Brennan PJ, Rado E, Convit J, Bloom BR. Lymphocyte suppression in leprosy induced by unique $M$. Leprae glycolipid. Nature 1984 ; 308 : 194.

9. Nath I. Reversal of T cell corresponsiveness in lepromatous leprosy. Lepr Rev 1986 ; 57 : 207-12.

10. Kaplan G, Weinstein DE, Steinman RM, et al. An analysis of in vitro $T$ cell responsiveness in lepromatous leprosy. $J$. Exp Med 1985 ; 162 : 917-29. 11. Lagrange $P H$, Hurtrel $B$. Induction of protective immunity to mycobacterial infections. Lepr Rev 1986 ; 57 (suppl. 2) : 231-44. $m / s \quad n \circ$ vol. 3, octobre 87 
des (Dasypus novimcinctus) entraîne, en 18 à 24 mois, dans 30 à $50 \%$ des cas, une maladie généralisée, ressemblant à la forme lépromateuse humaine. Une quantité impressionnante de bacilles de Hansen peut alors être récoltée dans les tissus infectés (par exemple : $10^{10}$ bacilles par gramme de tissu). Ce dernier modèle, qui permet donc de recueillir de grandes quantités de bactéries viables, a été à l'origine de l'essor des études biochimiques, ultrastructurales, immunologiques actuelles. Il a entraîné le développement par l'OMS d'un programme de recherches devant aboutir à la production d'un vaccin spécifique antilèpre. L'isolement et la caractérisation d'antigènes recombinants à partir des banques génomiques d'ADN de Mycobacterium leprae [5] semblent cependant aujourd'hui les méthodes les plus prometteuses.

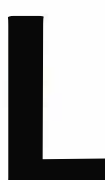

a deuxième particularité de la lèpre concerne les immunologistes : l'expression pathologique de la maladie, variable suivant les individus et parfois chez le même individu, est directement liée aux réponses immunitaires développées par l'hôte. Ainsi, les différentes formes de la maladie s'inscrivent suivant un spectre immunitaire continu, parfaitement intégré, qui est à la base de la classification faite par Ridley et Jopling [6]. Deux pôles extrêmes, fixes chez un même individu, sont très bien décrits. (a) Le premier est appelé lépromateux polaire (LLp): il correspond à une absence complète de réponse immunitaire cellulaire spécifique à $M$. leprae (in vitro et in vivo). Ceci est associé à une non limitation de la multiplication de $M$. leprae dans les lésions cutanées, muqueuses et nerveuses. Deux cellules prédominent dans ces lésions, des macrophages remplis de bacilles associés, sous forme de "globi " (cellules de Virchow) et des lymphocytes suppresseurs CD8. (b) Le deuxième pôle est appelé $\mathrm{m} / \mathrm{s} n^{\circ} 8$ vol. 3 , octobre 87 tuberculoïde (TT) car il réalise un granulome épithélioïde, où prédominent les lymphocytes CD4 et des cellules monocytaires dérivées du sang circulant. La réponse locale cellulaire spécifique est intense et aucun bacille n'est retrouvé localement.

Les formes intermédiaires, appelées borderline, peuvent évoluer, soit vers le pôle lépromateux (forme borderline lépromateuse ou forme lépromateuse subpolaire) soit vers le pôle tuberculoïde (forme borderline tuberculoïde). Les mécanismes sous-jacents de cette variabilité des formes borderline ne sont pas connus avec certitude. Plusieurs mécanismes, parfois contradictoires, ont été avancés, en particulier afin d'expliquer l'absence de réponse cellulaire spécifique des formes lépromateuses. Le premier concernait la mise en évidence de cellules lymphocytaires CD8 à fonctions suppressives qui sont stimulées spécifiquement par le glycolipide phénolique 1 (GLP1), antigène spécifique de $M$. leprae découvert par Brennan et al. [7] et qui est actuellement utilisé pour le diagnostic sérologique [8]. Plus récemment, il est apparu que l'impossibilité des cellules CD4 à répondre aux antigènes de $M$. leprae était due à l'absence de production d'IL2 par ces cellules [9]. Enfin, Kaplan et al. [10] ont bien démontré l'hétérogénéité des non répondeurs à $M$. leprae : parmi les sujets présentant des formes multibacillaires, certains semblent être dépourvues de cellules CD4 répondant à $M$. leprae ainsi que de cellules suppressives (macrophage ou lymphocyte CD8) ; d'autres sujets sont au contraire capables, en présence d'IL2 exogène, de répondre aux antigènes de $M$. leprae. Les premiers malades sont ainsi des non répondeurs par absence totale de cellules reconnaissant les antigènes de $M$. leprae et d'autres mycobactéries (ex : PPD); les seconds sont de faibles répondeurs dont la réponse est supprimée par un mécanisme peut-être non univoque (lymphocytes $\mathrm{T}$ suppresseurs, macrophages suppresseurs).

Le développement des techniques de clonage des cellules $T$, de production d'antigènes recombinants et la constitution de banques d'anticorps monoclonaux, ainsi que d'anticorps antiidiotypiques devraient permettre de mieux analyser et caractériser les épitopes importants dans l'induction et la régulation de la réponse cellulaire immunitaire à $M$. leprae, et ainsi de mieux comprendre le déterminisme des formes d'expression de la lèpre. Ces données devront être intégrées à celles de l'immunogénétique [11]. On pense maintenant que la plus ou moins grande susceptibilité à développer la lèpre serait contrôlée par un gène récessif non lié au complexe majeur d'histocompatibilité $(\mathrm{CMH})$. En revanche, lorsque la maladie est déclarée, son expression semble être contrôlée par un ou plusieurs gènes liés au $\mathrm{CMH}$ impliquant les molécules de classe II (DR, DQ).

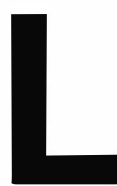

a troisième particularité de la lèpre concerne les épidémiologistes. En effet, les formes cliniquement identifiables ne représentent qu'un faible pourcentage de la morbidité potentielle, du fait de l'existence de formes infracliniques, impossibles actuellement à diagnostiquer. Après une période d'incubation très longue (de 5 à 10 ans), ces formes évoluent dans leur grande majorité vers les formes lépromateuses multibacillaires et sont potentiellement contaminantes pendant la période asymptomatique. Le développement de tests sérologiques simples, fiables et bon marché, utilisant le GLP1 et la mise au point de tests détectant les antigènes spécifiques devraient rapidement permettre de mieux cerner la morbidité réelle de la maladie. Cette approche pourrait alors faciliter la réalisation d'une stratégie cohérente de la lutte contre la transmission des bactéries pathogènes et donc entraîner l'extinction de cette maladie millénaire 